\title{
Resolution enhancement of ultrasonic B-mode images
}

\author{
S A Mosey, P C Charlton and I Wells
}

Submitted 14.09.12

Accepted 19.12 .12

\begin{abstract}
The use of ultrasonic array imaging systems is now commonplace throughout the NDT industry, but interpretation of the images produced by such systems can be a time-consuming process, which is further complicated by the inherent suboptimal resolution of such images. This paper proposes a three-step processing chain: noise-tosignal ratio (NSR) deconvolution, beam model weighted synthetic aperture focusing technique (BMW-SAFT) and statistical background noise removal (SBNR) image noise removal, which can be used to significantly enhance the temporal and spatial resolution of these systems. A validated simulation model is used to generate B-scan image data. Conventional Wiener filter deconvolution and SAFT methods are compared to those proposed, with results indicating a significant improvement.
\end{abstract}

\section{Introduction}

The signal received from an ultrasound transducer contains information about the component on which the transducer is placed. When the amplitude of this signal is plotted on a colour scale against transducer position, a cross-section representation of the component is created; these are called B-scan images. B-scan images are an extremely useful tool to the NDT technician,

Stephen Mosey is currently employed as a Senior Research Associate at Swansea Metropolitan University. Prior to this, he worked for two years at Silverwing UK and for seven years at Inspection Solutions (Cymru) Ltd as a design engineer. He was awarded a BSc in electrical and electronic engineering from the University of Glamorgan in 2003; he is also studying for a PhD. His areas of interest are digital signal processing, ultrasonic instrumentation and high-level ultrasonic software design. Tel: +44 (0)1792 481000; Email: stephen.mosey@smu.ac.uk

Dr Peter Charlton is currently employed as a Senior Research Fellow in NDT and is also the Course Director for the MSc in NDT at Swansea Metropolitan University. He was previously the Managing Director for Inspection Solutions (Cymru) Ltd. His areas of interest are numerical modelling, digital signal processing, electromagnetic and ultrasonic methods of NDT. Tel: +44 (0)1792 481000; Email: peter.charlton@smu. ac.uk

Professor Ian Wells obtained a BSc in Physics from Manchester University, Manchester, UK, in 1982, an MSc in Microelectronics and Communications Systems from the University of Westminster, London, UK, in 1986 and a PhD in Signal Processing Architectures for Speech Recognition from the University of the West of England, Bristol, UK, in 1996. He is currently Professor of Digital Signal Processing and the Assistant Dean of the Faculty of Applied Design and Engineering at Swansea Metropolitan University, Swansea, UK, where he has worked since 1996. He was previously the Research Director of Technium Digital, University of Wales Swansea, Swansea, UK, which is a high-technology incubator unit. He also worked as an electronic engineer for AB Electronics, South Wales, UK, after completing his first degree. Professor Wells is also a Fellow of the Institution of Engineering and Technology (FIET), a Senior Member of the Institute of Electrical and Electronic Engineering (SMIEEE), a Fellow of the British Computer Society (MBCS) and a Chartered Engineer (CEng). allowing them to 'see' inside a component. B-scan images can be created by measuring the distance a single transducer has moved, or by using multiple element linear array transducers. As with many imaging systems, ultrasonic systems are not without their problems. As a consequence of the way sound waves propagate and behave, the resolution of the system will always be suboptimal The oscillations of the crystal in the transducer create a wavelet, which contaminates the echo signals from the material under test. Also, due to beam divergence, echoes will be received from targets within the material under test that are not directly in the line of sight of the transducer. Due to this, the images created tend to exaggerate feature sizes and appear blurred.

This paper proposes a three-step scheme of signal processing measures to increase the resolution of these images. The first step uses a deconvolution method to improve the temporal or through-thickness resolution of the acquired images. The proposed deconvolution method uses a threshold to determine safe areas within the spectra of the signals to ensure stability. The second step uses a new BMW-SAFT to improve the spatial resolution in the scan axis of the images. The BMW-SAFT algorithm uses the Fraunhofer approximation to model the far field of the ultrasound beam, which is then used to determine the size of the aperture and the weights applied across the aperture during the focusing process. The final step utilises a statistical method to remove background speckle noise within the images. Speckle noise can come from many different sources, but it is noted that after the first step of the proposed process, the deconvolution method creates speckle noise within the image as a side effect. To test the methods proposed in this paper, a validated computer simulation model has been developed to generate single A-scans and entire B-scans. The simulation model will be described in Section 2. Section 3 describes the three different processing methods proposed by this paper and compares them to their more conventional counterparts. In Section 4 , a discussion on the performance and suitability of these methods is provided.

\section{Simulation model}

\subsection{Forward model}

An ultrasonic inspection system can be considered to be linear time invariant. The transfer function for the material under test can be considered as a series of Dirac delta functions. Each Dirac delta function will represent a feature within the part under test. These features will include any interfaces where there is a change in acoustic impedance, such as the far surface of the part, any defect indications or reflections caused by the grain structure of the material, see Figure 1.

The amplitude of the reflected signal $y(t)$ will depend upon the difference in the acoustic impedance of the two adjoining materials and the amplitude of the transmitted incident wave, as shown in Equation (1):

$$
R E=\left(\frac{Z_{1}-Z_{2}}{Z_{1}+Z_{2}}\right)^{2} \times A_{i}
$$

where $Z_{1}$ and $Z_{2}$ are the acoustic impedances of the two media and $A_{i}$ is the amplitude of the incident wave. 


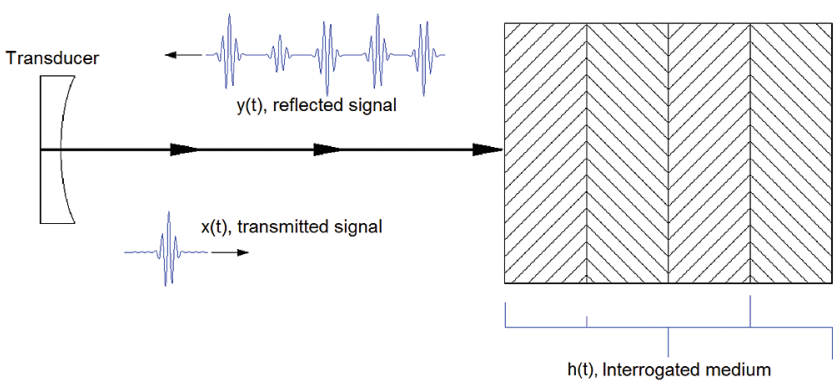

Figure 1. Reflected signals from an interrogated medium ${ }^{[1]}$

For each reflector within the inspected media, the apparent depth needs to be calculated for each transducer position along the surface of the material. As illustrated in Figure 2, this is achieved using a simple Pythagorean method, where the apparent depth, $r$, of the current indication, $P_{x, y}$, is given by Equation (2). $P_{x}$ and $P$ are the coordinates of the reflector and $X_{n}$ is the location of the transducer along the surface of the material:

$$
r=\sqrt{P_{y}{ }^{2}+\left(X_{n}-P_{x}\right)^{2}}
$$

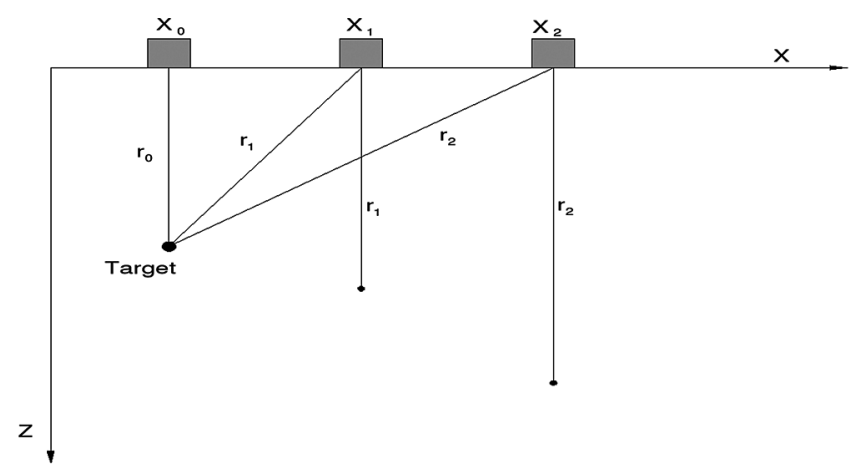

Figure 2. Apparent depth of reflector from different transducer positions

The process of determining the apparent depth must be performed for each indication within the material. At these locations, a reflection coefficient can be calculated based upon the distance from the transducer, the attenuation characteristics of the material and the shape of the ultrasound beam from the transducer.

\subsection{Attenuation}

Many materials exhibit anisotropic attenuation characteristics. This is taken into account in Equation (3), where $\alpha_{x}$ and $\alpha_{y}$ are the scalar attenuation coefficients of the material and $\Delta_{x}^{x}$ and $\Delta_{y}^{y}$ are the distance from the transducer to the reflector in $x$ and $y$, respectively:

$$
\alpha_{\text {total }}=\alpha_{x}{ }^{2 \Delta x} \alpha_{y}{ }^{2 \Delta y}
$$

Material attenuation is the combination of the absorption and scattering of the ultrasound beam in the material. Material attenuation can be estimated by measuring amplitudes from a series of reflectors positioned at different depths, plotting these amplitudes against their depth and fitting an exponential curve to the points. The attenuation coefficient can be derived from the equation for the amplitude-depth curve, as shown in Figure 3. For the material used in the validation section, cold rolled steel, the compression wave velocity was found to be $5915 \mathrm{~ms}^{-1}$ and the attenuation coefficients were determined experimentally as $-0.023 \mathrm{~dB} / \mathrm{mm}$ parallel to the rolling direction and $-0.018 \mathrm{~dB} / \mathrm{mm}$ perpendicular to the rolling direction.

\subsection{Beam model}

Ultrasonic beam modelling can be done using any one of several different methods; Huygens' method provides accurate information about the entire beam produced by a transducer at the expense of a

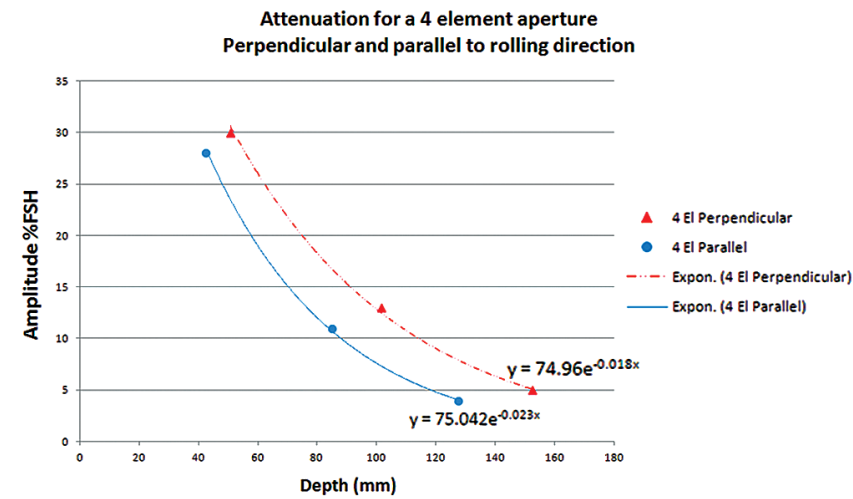

Figure 3. Experimental results to determine material attenuation

large computation time. Various other methods have been proposed; far-field approximations such as the Fraunhofer approximation are only valid in the far field of a transducer as they are based on the Fourier transform of the aperture:

$$
a(\theta)=\left\{\left(a_{0} w \frac{\sin \alpha}{\alpha}\right)\left(\frac{\sin (N \beta)}{\sin (\beta)}\right)\right\}^{2}
$$

For expediency, the Fraunhofer approximation is the beam model adopted for this paper, and given in Equation (4), where $\alpha=\frac{k w \sin \theta}{2}, \beta=\frac{k d \sin \theta}{2}, a_{0}$ is the initial amplitude, $w$ is the element width, $d$ is the element pitch, $N$ is the number of active elements in the aperture, $k$ is the wave number given by $k=\frac{2 \pi}{\lambda}$ and $\theta$ is the angle at which the beam profile is to be calculated, relative to the transducer normal. This equation can easily be altered to use the Cartesian coordinate system by replacing $\theta$ with $\tan ^{-1} \frac{x}{z}$, where $x$ and $z$ are the lateral and axial distances from the transducer to the point in question.

The value for attenuation is then multiplied by the point of the beam profile at which the reflector lies relative to the transducer. This number gives the reflection coefficient to be inserted at the apparent depth for the indication. This operation yields a bitmap containing the locations and amplitudes where the echoes will appear.

\subsection{Convolution}

The observed signal from an ultrasonic measurement system, $y(t)$, can be expressed as the convolution of the response of the measurement system (wavelet) $x(t)$ with the transfer function of the material $h(t)$, summed with random noise $\varepsilon(t)^{[2]}$ :

$$
y(t)=(h(t) \otimes x(t))+\varepsilon(t)
$$

The wavelet used in the simulation model can be calculated simply by placing a Gaussian envelope over a sine wave at the probe's fundamental frequency, as illustrated in Equation (6) and Figure 4:

$$
x=\sin \left(\left(\frac{2 \pi}{\lambda}\right)(d-v t)\right) \cdot e^{\frac{-(d-v t)^{2}}{p w^{2}}}
$$

where $x$ is the resulting wavelet, $\lambda$ is the wavelength in the material under test, $d$ is the depth into the material, $v$ is the velocity of sound through the material, $t$ is the position of the wavelet along the time or distance axis and $p w$ is the pulse width. Convolving the bitmap of reflection coefficients with the wavelet, using Equation (5), will yield a noiseless B-scan image. White Gaussian noise (WGN) can now be added to this, simulating random electrical noise, to create a more realistic simulation. To simulate material noise, a number of lowamplitude reflectors must be scattered randomly through the bitmap. A flowchart for the simulation model can be seen in Figure 5. 


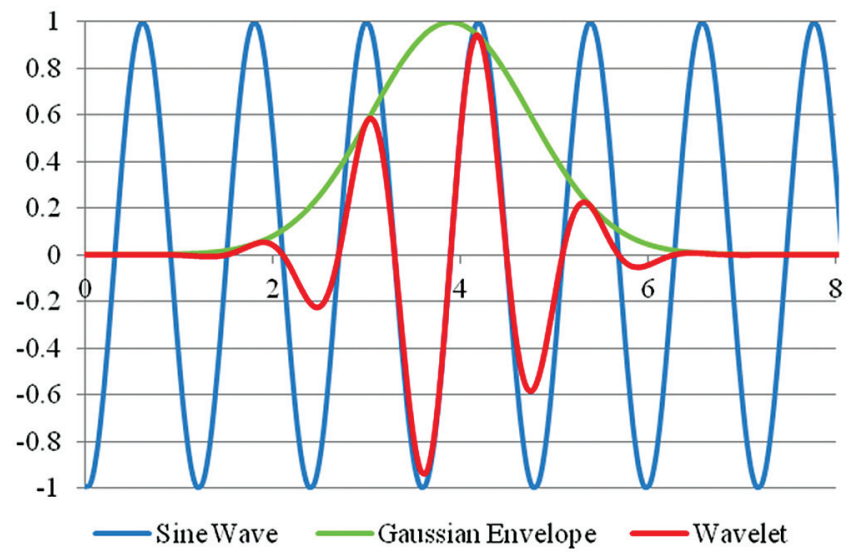

Figure 4. Wavelet simulation using a sine wave multiplied by a Gaussian envelope

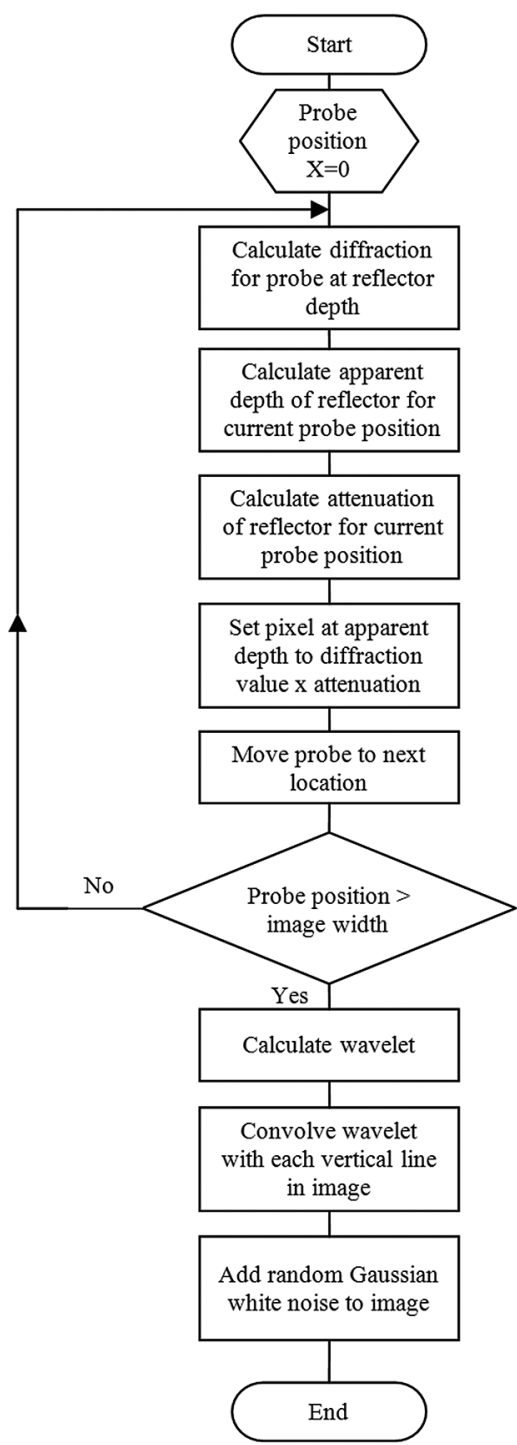

Figure 5. Flowchart describing the proposed simulation model

\subsection{Simulation validation}

The simulation method was validated by comparing measurements of amplitude and depth taken from images of side-drilled holes captured using a GE Phasor XS ultrasonic phased array instrument with simulated images of the same. The capture system used had a 32-element transducer with no wedge. Measurements were taken of the peak amplitude and position of the echoes within the image using a gate across the A-scan. These measurements were plotted against element position in $\mathrm{mm}$.
The simulation method described here provides a quick means of simulating simple models where only isolated indications are considered. A comparison of measured and simulated amplitudes and depths can be seen in Figure 6. Deviations in the measured amplitude from the simulated amplitude could be attributed to one of many sources, such as couplant between the transducer and the material under test or pressure on the transducer. The experimental measurements of depth were only possible in the region directly around the indication in the image; this was due to the amplitude of the signal falling below the height of the gate used on the A-scan and in to the background noise of the A-scan, making measurements impossible.

\section{Processing methods}

\subsection{Deconvolution}

Deconvolution can be performed in one of many ways. The time domain solution, as shown in Equation (7), is unstable as it relies on the first number in the wavelet, $x(0)$, being non-zero - this is seldom the case:

$$
\begin{gathered}
h(0)=\frac{y(0)}{x(0)} \\
h(n)=\frac{y(n)-\sum_{k=0}^{n-1} h(k) x(n-k)}{x(0)}, n \geq 1
\end{gathered}
$$

Convolution in the time domain can be expressed as a multiplication in the frequency domain, so it follows that deconvolution must be a divide in the frequency domain. This can, again, cause problems of stability as ultrasonic signals are inherently band limited and, outside the bandwidth of the signals, the spectra of both the observed signal and the wavelet approach zero.

The ideal image will only contain information from $h(t)$; to do this contributions from $x(t)$ must be removed from the signal. Sin and $\mathrm{Chen}^{[3]}$ compared several deconvolution methods, including Wiener filtering, a combination of Wiener filtering with autoregressive spectral extrapolation and minimum variance deconvolution (MVD), which used a Kalman filter. They concluded that a form of Wiener filter was most efficient. While a Wiener filter used in isolation has the advantage of being simpler than other methods, it is ill-conditioned to the problem due to the band limited nature of ultrasonic pulses ${ }^{[4]}$. To improve deconvolution, the useful bandwidth of the signals must be maximised to ensure the optimal result is achieved.

For a frequency domain deconvolution to be successful, the division must only occur within the bandwidth of the signals. The optimum value of the threshold is related to the signal-to-noise ratio of the observed signal: the more noise present in the signals, the higher the threshold needs to be. For this reason, the proposed deconvolution method uses a threshold based on the noise-to-signal ratio (NSR). The NSR is calculated by the ratio of the average amplitude of all the sample points in the spectrum of the observed signal that are in the same frequency space as sample points that are below a threshold $T_{2}$ in the wavelet spectrum, to the peak value of the observed signal's spectrum. This is illustrated in Equation (8) and Figure 7:

$$
N S R=\frac{\frac{1}{\#\left(\left|X_{\omega}\right| \leq T_{2}\right)} \sum_{n=1}^{N}\left|Y_{\omega n}\right| \cdot \begin{cases}1, & \left|X_{\omega n}\right| \leq T_{2} \\ 0, & \left|X_{\omega n}\right|>T_{2}\end{cases} }{\max \left|Y_{\omega}\right|}
$$

where $T_{2}$ is the threshold given by $T_{2}=\max \left|X_{\omega}\right| \cdot 10^{T_{1 / 20}}, N$ is the length of the two signals, $Y_{\omega}$ is the spectrum of the observed signal, $X_{\omega}$ is the spectrum of the wavelet and the \# symbol is used to determine the cardinality of the parenthesised expression it precedes. 


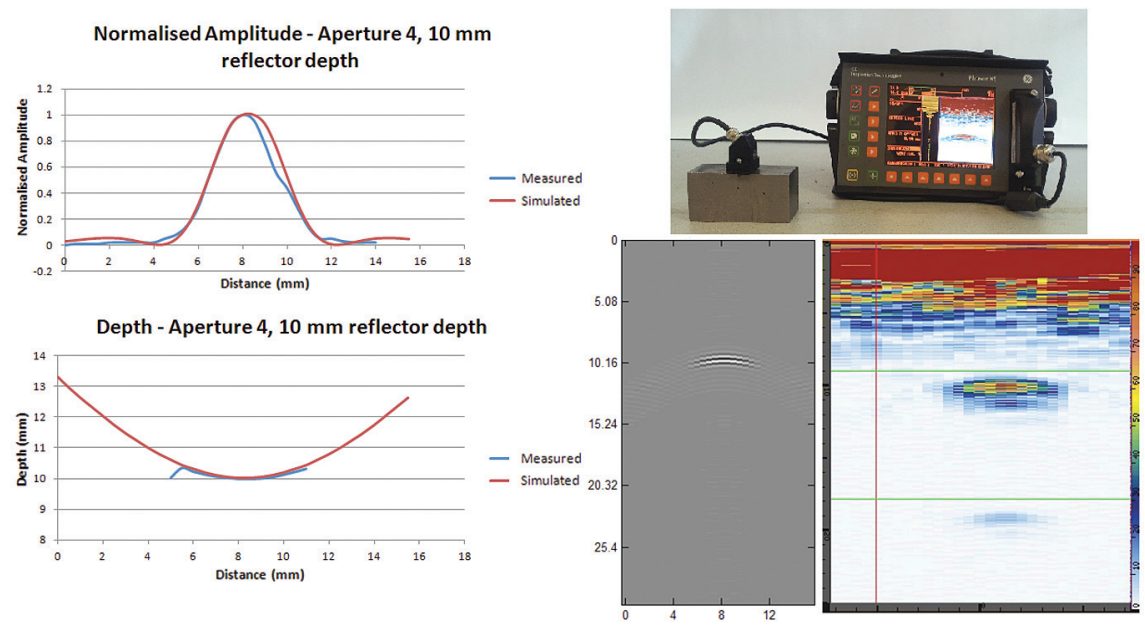

Figure 6. Top left: comparison of normalised amplitude for simulated and measured image of $1 \mathrm{~mm}$ side-drilled hole at a depth of $10 \mathrm{~mm}$ from the surface; bottom left: comparison of simulated and measured depth from $1 \mathrm{~mm}$ side-drilled hole; top right: experimental set-up; bottom centre: simulated results; bottom right: measured results $x_{\omega}$

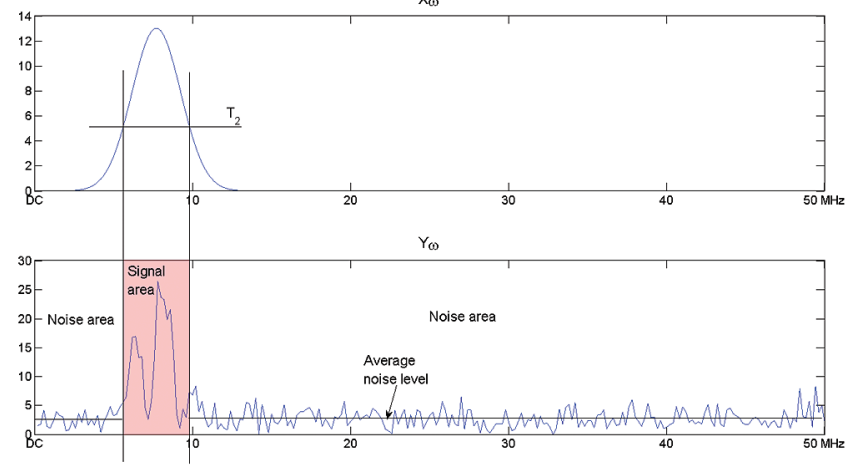

Figure 7. Selection of noise and signal areas for NSR calculation

The estimation of NSR is now used to create the threshold $T_{3}$, which is used to determine the safe areas of the signal where stable deconvolution can occur. Outside the safe areas, the estimate of the material impulse response spectrum is set to zero, while inside the safe areas it is the ratio of the observed signal spectrum to the wavelet spectrum, as shown in Equation (9):

$$
\hat{H}_{\omega}=\frac{Y_{\omega}}{X_{\omega}} \cdot \begin{cases}1, & \left|X_{\omega}\right| \geq T_{3} \\ 0, & \left|X_{\omega}\right|<T_{3}\end{cases}
$$

where $T_{3}=N S R \cdot \max \left|X_{\omega}\right|$ and $\hat{H}_{\omega}$ is an estimate of the spectrum of the reflection coefficients of the material under test. The time domain estimate of the reflection coefficients of the material under test can now be calculated by taking the inverse Fourier transform of $\hat{H}_{\omega}$.

As an illustration, the results of a NSR deconvolution for a typical system can be seen in Figure 8. This shows the original

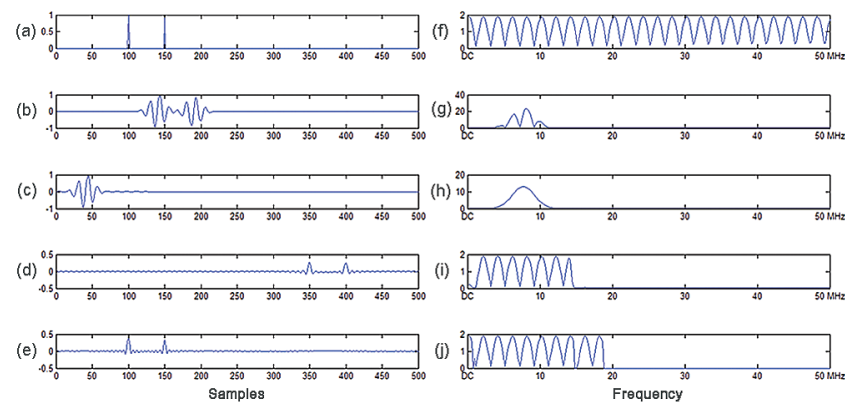

Figure 8. (a) Reflection coefficients of material under test; (b) simulated A-scan based on reflection coefficients in (a) and wavelet in (c); (c) wavelet simulated using a Gaussian envelope over a sine wave; (d) result from Wiener filtering the A-scan in (b); (e) result from NSR deconvolution of A-scan using a threshold $T_{1}$ of $-60 \mathrm{~dB}$; (f) to (j) the spectra of (a) to (e), respectively impulse response of the material, the synthetic A-scan and the results of Wiener deconvolution and NSR deconvolution. The impulses can be seen in the wrong place in the result of the Wiener filter, but their relative amplitudes are correct. In the results from the NSR deconvolution, the impulses have the correct position and relative amplitude as the original impulses, while being sharper than the Wiener result. This can be seen clearly in the spectra in Figures 8(i) and (j), where the spectrum for the Wiener filtered A-scan has a shorter bandwidth than that of the NSR deconvolved A-scan.

While deconvolution has the ability to increase the temporal or A-scan resolution of an ultrasonic inspection system, it is incapable of altering a system's spatial resolution along an array's scan axis. To improve the spatial resolution of an ultrasonic imaging system, focusing algorithms such as SAFT must be employed.

\subsection{Synthetic aperture focusing technique}

\subsubsection{Conventional SAFT}

The synthetic aperture focusing technique (SAFT), originally developed for use in radar systems ${ }^{[5]}$, has the ability to increase the focus of a B-scan image, removing tails from indications within the image. SAFT uses a delay and sum approach to focusing, adding the pixel intensities of all the pixels with indications that could have originated from the currently focused pixel. This is illustrated in Equation (10):

$$
P^{\prime} x, y=\frac{1}{m} \sum_{i=-\frac{m}{2}}^{\frac{m}{2}} P_{x+i, \sqrt{i^{2}+y^{2}}}
$$

where $P^{\prime}$ is the resulting focused image, $m$ is the aperture size, $P$ is the original image and $x$ and $y$ are coordinates within the images. For a given pixel within the image, all the pixels along a parabola are averaged with the result placed at the apex of the parabola in the new image.

In 1984, Burch and Burton ${ }^{[6]}$ proposed that a weighting factor be applied to the SAFT algorithm to reduce the effect caused by side lobes. They experiment with standard signal processing windowing functions to weight the algorithm. The width of the synthetic aperture was calculated based on the beam width at the required depth of focus. The lengths of slot-type indications in simulated data, and the amplitude of the spurious side lobes, were measured before and after processing. They found that for the majority of cases a Gaussian function falling to 0.3 at the extremities gave the best results.

\subsubsection{Proposed beam model weighted SAFT algorithm}

Further to Burch and Burton's work, a new beam model weighted synthetic aperture focusing technique (BMW-SAFT) is presented. The Gaussian function used by Burch and Burton is of a similar shape to the actual beam profile produced by an ultrasonic transducer; they also allude to the optimum aperture width being closely related to the width of the ultrasonic beam at the required focal depth. An obvious step would be to create a weighting function based on the amplitude distribution of the beam and vary the aperture width with focal depth based on the beam width of the transducer at that depth. The beam intensity of a transducer can be calculated in several ways: the Huygens-Fresnel method can provide information about the near field but can be quite time consuming to compute; while the Fraunhofer approximation gives a good representation of, and is only valid in, the far field of a transducer, though it is 
quicker to calculate. The algorithm for BMW-SAFT is illustrated in Equation (11) and Figure 9, where $m_{y}=\#\left(W_{x z} \geq T\right), W_{x z}$ is the weighting function created from the Fraunhofer approximation of the transducer's beam profile (Equation (4)) by replacing the $\theta$ component of $\alpha$ and $\beta$ with the Cartesian representation as described previously, and $T$ is a threshold to determine the extent of the beam to be used. For example, $T$ could be calculated based upon a beam angle as illustrated in Figure 10.

$$
P^{\prime} x, y=\frac{1}{m_{y}} \sum_{i=-\mathbf{m}_{y} / 2}^{\mathbf{m}_{y} / 2} P_{x+i, \sqrt{i^{2}+y^{2}}} \cdot W_{i, \sqrt{i^{2}+y^{2}}}
$$

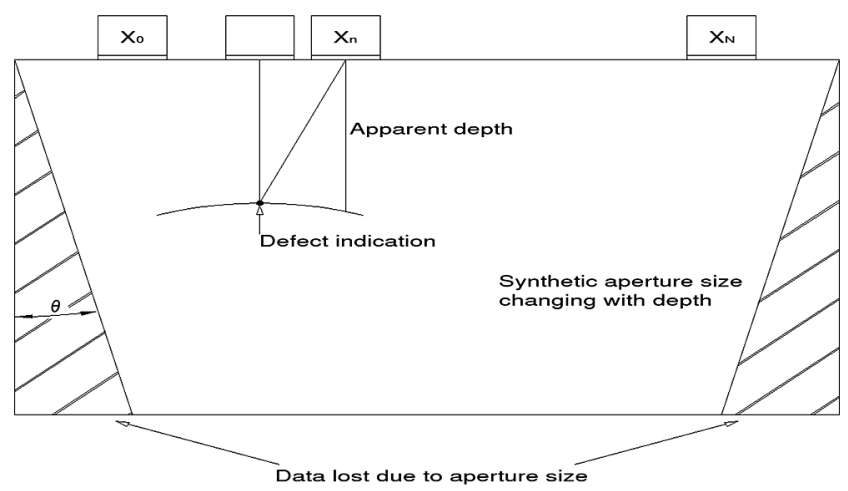

Figure 9. BMW-SAFT

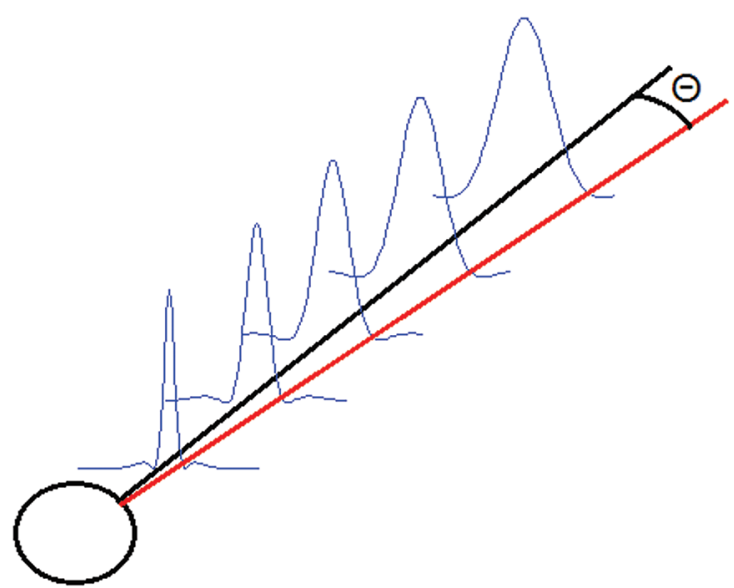

Transducer face

Figure 10. $T$ based on a beam angle

\subsection{Statistical background noise removal (SBNR)}

Images processed by deconvolution can exhibit higher levels of background speckle noise ${ }^{[7]}$; this is due to the Fourier transforms of the deconvolution process. A scheme for background noise removal was proposed previously ${ }^{[8]}$. Before the SBNR algorithm can be applied, the data must first be rectified. This is achieved through the use of the Hilbert transform. The image is now ready to be processed by the SBNR algorithm. The algorithm is an iterative process. It first calculates a mean A-scan for the image using one of two methods, depending upon whether a training zone is being used to create the mean A-scan (Equation (12)), or if the entire image will be used (Equation (13)). If a training zone is to be used, the operator must specify a defect-free region of the image to allow the algorithm to calculate a mean A-scan:

$$
\overline{\operatorname{AScan}(y)}=\frac{\sum_{x=L}^{R} \operatorname{AScan}(x, y)}{(R-L)}
$$

where $R$ is the right-most point of the training zone, $L$ is the leftmost point of the training zone and $x$ and $y$ are the coordinates in the image:

$$
\overline{\operatorname{AScan}(y)}=\frac{\sum_{x=0}^{N-1} A \operatorname{Scan}(x, y)}{N}
$$

where $N$ is the total number of A-scans in the image. This mean A-scan is then sequentially subtracted from each A-scan in the image (Equation (14)). By subtracting a mean A-scan, common features (such as the backwall echo and any general noise) in the image will be removed. The image is then normalised; this has the effect of increasing the contrast in the image. After the image has been normalised, the mean standard deviation is calculated; this enables us to determine the mean spread of data within the image (Equation (15)). The whole process is then repeated until the rate of change of mean standard deviation is lower than a predetermined threshold.

$$
\begin{aligned}
& \operatorname{AScan}(y)^{\prime}=\operatorname{AScan}(y)-\overline{\operatorname{AScan}(y)}
\end{aligned}
$$

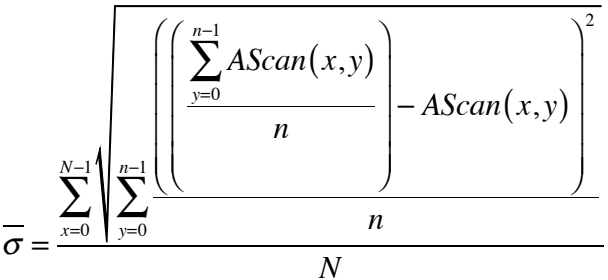

where $\bar{\sigma}$ is the mean standard deviation, $N$ is the number of A-scans in the image, $n$ is the number of samples in each A-scan and $x$ and $y$ are coordinates in the image. By definition, the standard deviation is the root mean square of the deviation of all the samples from the mean. Equation (15) can be derived from the standard deviation formula, as shown in Equation (16):

$$
\sigma=\sqrt{\frac{\sum_{i=1}^{n}(\bar{x}-x)^{2}}{n}}
$$

\section{Results}

A simulated image of five point reflectors was imaged using a 7.5 MHz transducer with an aperture of four elements, each element being $0.49 \mathrm{~mm}$ wide with a $0.5 \mathrm{~mm}$ element pitch. The experimentally derived attenuation coefficients from the simulation validation were used and the compression wave velocity used was $5915 \mathrm{~ms}^{-1}$. WGN with an amplitude of $\pm 15 \%$ was added to the image. The simulation output can be seen in the first image in Figure 11. Although this simulation does not take into account the shadowing of more distant reflectors that might be masked by reflectors nearer to the transducer, this does enable us to compare each indication in isolation. This will be important in assessing the performance of the two SAFT processed images. The second image shows the output from the proposed NSR deconvolution method. It can clearly be seen that the background noise has increased notably from the original data, while the ringing of the pulses has been reduced, thereby increasing the temporal resolution of the image. The third and fourth images show the results from the conventional SAFT processed NSR deconvolved data and the results of the BMW-SAFT processed NSR deconvolved data. The conventional SAFT image was processed with an aperture size such that optimal focusing was achieved near the top-most indication. In the conventional SAFT processed image, it can be seen that the focusing performance of the algorithm deteriorates with depth for a fixed aperture size, while the BMW-SAFT processed image has a more uniform performance with depth. The conventional SAFT image also suffers from artefacts, which can be seen either side of the indications near the bottom of the image. These artefacts take the form of arcs originating at the indication's centre and extending outwards from the top and bottom towards the sides of the image. This is another anomaly that is present in the conventional SAFT processed image but not in the BMW-SAFT processed image. In 

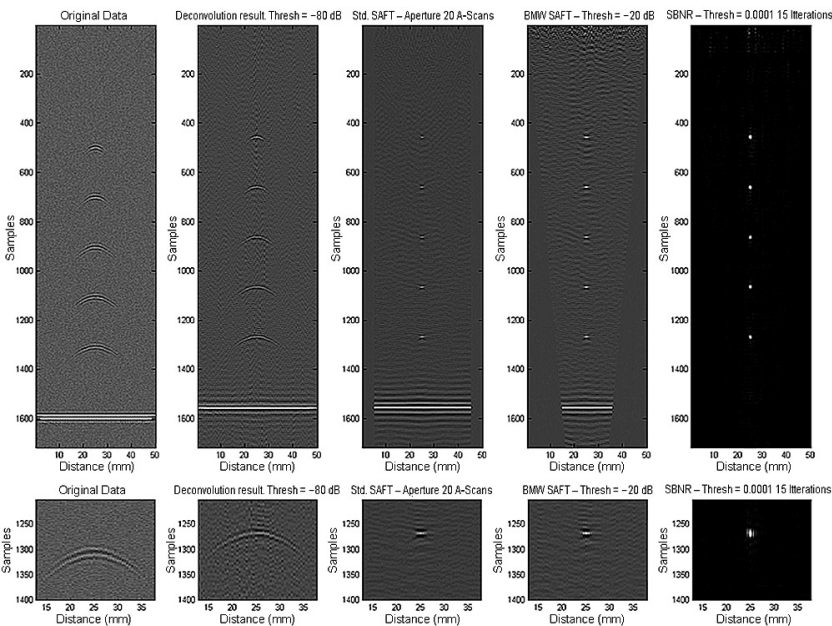

(a)

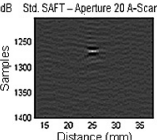

(c)

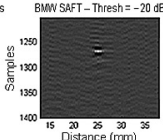

(d)

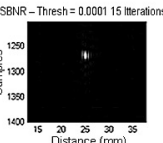

(e)

Figure 11. (a) Original simulated image; (b) NSR deconvolved image (threshold = $-80 \mathrm{~dB}$ ); (c) NSR deconvolved and conventional SAFT processed image (aperture $=20 \mathrm{~A}$-scans); (d) NSR deconvolved and BMW-SAFT processed image (threshold $=-20 \mathrm{~dB}$ ); (e) NSR deconvolved BMW-SAFT and statistical background noise removal processed image (SBNR threshold $=0.001-12$ iterations); with an enlarged area from each below

the final image, the result of the SBNR algorithm can be seen. The SBNR algorithm has successfully enhanced the contrast of the image while reducing the speckle noise. A-scans through the indications from each of the images can be seen in Figure 12. This shows more clearly the uniformity of the BMW-SAFT algorithm, as the echo amplitudes from the indications can be seen to be more or less equal.

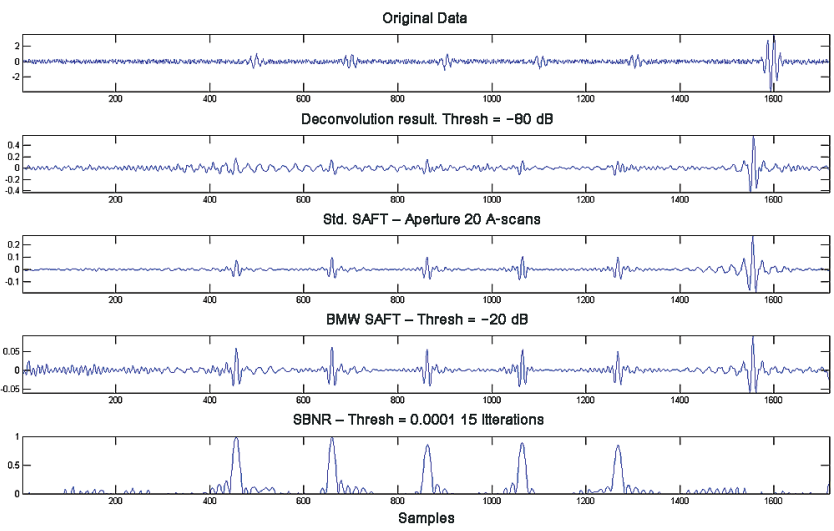

Figure 12. A-scans taken through indications from Figure 11. Top to bottom: original data; NSR deconvolved A-scan; conventional SAFT processed A-scan; BMW-SAFT processed A-scan; and SBNR processed A-scan

\section{Comments and conclusions}

The simulation method presented provides a rapid means of simulating ultrasound B-scan images. Although this method currently only supports a $0^{\circ}$ inspection angle, it could be modified to include other beam angles. The simulations in this paper were performed in a matter of seconds on a modest desktop computer; simulation time depends primarily upon the number of indications within the image. Deconvolution can provide a means of sharpening pulses, increasing the temporal resolution of the signals. When working with simulated images, estimation of the wavelet becomes moot, conversely with real data some estimation of the wavelet would be required. Wavelet estimation can be done in several ways: an estimation of the pulse duration and frequency could lead to simulating a wavelet using the method described; or if that is not possible, a signal from an isolated indication from an image could

be used. The proposed NSR deconvolution method provides better results than the Wiener filter, although for the NSR deconvolution a suitable threshold for calculating the noise-to-signal ratio must be determined. While several variations of the SAFT algorithm have been proposed by many, the proposed BMW-SAFT algorithm includes information about the ultrasonic beam used to create the image. This enables the algorithm to sharply focus the entire image without any focal degradation with depth, as can be seen with the standard SAFT algorithm. Using modern computers, the increase in complexity of the algorithm is barely noticeable. The beam model used to create the weighting factors and aperture size illustrated in this paper uses a linear array of rectangular elements, but could easily be tailored to suit any transducer configuration. A linearly increasing loss of data near the edges of images processed with the BMW-SAFT process would need to be compensated for by acquiring extra data at the start and end of the scan. The SBNR algorithm used removes features common to all A-scans. This has the effect of removing the backwall echo, although in some situations this in undesirable, but for the application of looking at internal defects within a component, these indications are enhanced by the algorithm, simplifying their measurement.

\section{References}

1. C Chamzas, 'On the extrapolation of band-limited signals', $\mathrm{PhD}$, Polytechnic Institute of New York, 1980.

2. AN Sinclair, J Fortin, B Shakibi, F Honarvar, M Jastrzebski and M D C Moles, 'Enhancement of ultrasonic images for sizing of defects by time-of-flight diffraction', NDT\&E International, Vol 43, pp 258-264, April 2010.

3. S Sin and C Chen, 'A comparison of deconvolution techniques for the ultrasonic non-destructive evaluation of materials', IEEE Transactions on Image Processing, Vol 1, pp 3-10, January 1992.

4. F Honarvar, H Sheikhzadeh, M D C Moles and A N Sinclair, 'Improving the time resolution and signal-to-noise ratio of ultrasonic NDE signals', Ultrasonics, Vol 41, pp 755-763, 2004.

5. V Schmitz, S Chakhlov and W Muller, 'Experiences with synthetic aperture focusing technique in the field', Ultrasonics, Vol 38, pp 731-738, 2000 .

6. S F Burch and J T Burton, 'Ultrasonic synthetic aperture focusing using planar pulse-echo transducers', Ultrasonics, Vol 22, pp 275-281, 1984.

7. P Broberg, M Sjodahl and A Runnemal, 'Improved image quality in phased array ultrasound by deconvolution', presented at the 18th World Conference on Nondestructive Testing, Durban, South Africa, April 2012.

8. S Mosey, P C Charlton and I Wells, 'Iterative method for background noise removal in ultrasonic B-scan images', Insight, Vol 48, pp 677-681, November 2006. 